\title{
Estimation of the influence of the Nonequilibrium State of the Electronic Subsystem and the Dispersion Properties of a Solid-State Plasma Medium in the conditions of Decay Instability on the reflection of Laser Radiation
}

\author{
Maksym Iasechko ${ }^{1}$, Oleksandr Tymochko ${ }^{2}$, Oleksandr Kolodiazhnyi ${ }^{2}$, Volodymyr Larin ${ }^{3}$, Oleksandr Rybkin ${ }^{4}$, \\ Valerii Patalakha $^{5}$, Volodymyr Mazharov ${ }^{6}$, Nataliya Benyk ${ }^{7}$, Anastasiia Heilyk ${ }^{8}$, Mykhailo Mozhaiev ${ }^{9}$ \\ ${ }^{1}$ Doctoral Candidate, Ivan Kozhedub Kharkiv National Air Force University, Ukraine, \\ maxnik8888@gmail.com \\ ${ }^{2}$ Department of Air Navigation and Combat Control of Aviation, Ivan Kozhedub Kharkiv National Air Force University, Ukraine, \\ timochko.alex@gmail.com, kolodiazhnyi@gmail.com \\ ${ }^{3}$ Department of Application and Operation of Automated Control Systems, Ivan Kozhedub Kharkiv National Air Force University, Ukraine, \\ L_vv83@ukr.net \\ ${ }^{4}$ Department of Tactics and University Studies, Ivan Kozhedub Kharkiv National Air Force University, Ukraine, \\ rybakoff77@i.ua \\ ${ }^{5}$ Department Of The Anti-Aircraft Missile Troops, National Defense University of Ukraine named after Ivan Cherniakhovskyi, Ukraine, \\ valeryacad@gmail.com \\ ${ }^{6}$ Department of flight operations, aerodynamics and flight dynamics, Flight Academy of National Aviation University, Ukraine \\ mazharov@gmail.com \\ ${ }^{7}$ Department of Navigation and Ship Construction, State University of Infrastructure and Technology, Ukraine, \\ maxnik8888@gmail.com \\ ${ }^{8}$ Department of Higher and Applied Mathematics, State University of Infrastructure and Technology, Ukraine, \\ maxnik8888@gmail.com \\ ${ }^{9}$ Head of the Department, Hon. Prof. M. S. Bokarius Kharkiv Research Institute of Forensic Examinations, Ukraine, \\ mikhail.mozhayev@hniise.gov.ua
}

\begin{abstract}
The paper analyses the problem of protection of radio electronic means (REM) against electromagnetic (laser) terrorism, connected with the influence of laser radiation on REM, both of air and ground application, systems of various purposes. The lack of universal means of REM protection against laser radiation and the need to develop the effective means of protection, taking into account the energetic capabilities of electromagnetic terrorism, have been pointed out.
\end{abstract}

The impact of decay instability on the dispersive properties of the solid-state plasma media and the reflection of laser radiation have been estimated.

Key words: radio electronic means, electromagnetic radiation, ultrashort pulse duration, plasma protection technologies, gaseous plasma media.

\section{INTRODUCTION}

The analysis of the available literature and open information points to the problem of protecting REM, both of air and ground application, against the effects of electromagnetic (laser) terrorism, which is preconditioned by, on the one hand, the accessibility of special equipment to carry out electromagnetic terrorist attacks, and, on the other hand, the lack of universal means of protection of objects of impact.
There are known portable laser radiation generators, the use of which easily ensures not only the stopping of the car, burning all the electronics and the ignition system in it, but also the air means, completely destroying the electronic control and management systems, if it falls into the hands of a criminal or a terrorist.

Methods and ways of protection designed to date are not able to provide the required REM protection efficiency by their characteristics, primarily due to the absence of perfect case-screen (without defects), the impossibility of creating (the presence of structural openings, cracks, seams, connection points in enclosures, places for insertion of power cables, communication lines, etc.), or the use of sealed case-screens in relation to laser EMR (due to the presence of openings), the inability of existing limiters to withstand the impulse surges under the influence of a powerful EMR, insufficient performance and unacceptable oversized characteristics. It encourages the development and research of fundamentally new methods of REM protection against the destructive effects of laser EMR.

For the time being, special work is being done to counteract portable lasers, creating completely new means of protection using nature-like (plasma) technologies to protect REM from the influence of laser EMR, which allows the physical mechanism of reflection of laser EMR to be realized [1, 6, 9, 13-30]. 
The implementation of the physical mechanism of reflection of laser EMR from plasma solid-state material is possible due to the creation of conditions of decay instability in a protective case-screen at a sufficiently small magnitude of the field strength of the Langmuir wave.

It means that the solid-state plasma medium actually plays the role of a mirror and reflects the laser radiation in the opposite direction at a small thickness of the shielding material.

The aim of the article is to estimate the dispersion properties of a solid-state plasma medium in the reflection of laser radiation using the mechanism of decay instability.

\section{PROBLEM ANALYSIS}

According to the papers $[3,4,7,12]$, the creation of conditions of decay instability in a protective case-screen, in the form of a solid-state plasma medium, provides shielding of laser EMR as a sufficiently small magnitude of the field intensity of the Langmuir wave. It means that the solid-state plasma medium actually plays the role of a mirror and reflects the laser radiation in the opposite direction at a small thickness of the shielding material.

In the absence of the impact of laser EMR, the particle distribution over time due to relaxation become a Maxwellian one, which is described by a known expression [20]:

$$
f(v)=\left(\frac{m_{e}}{2 \pi T}\right)^{\frac{3}{2}} e^{-\frac{m_{e} v^{2}}{2 T}},
$$

where $\mathrm{T}$ is the thermodynamic air temperature;

$m_{e}-$ the mass of electron;

$\mathrm{V}-$ the velocity of particle flow.

The solution of this equation is provided in fig. 1, which shows that the particle distribution function evolves and sets the steady state $\mathrm{X}=\mathrm{E} / \mathrm{E}_{\mathrm{f}}$.

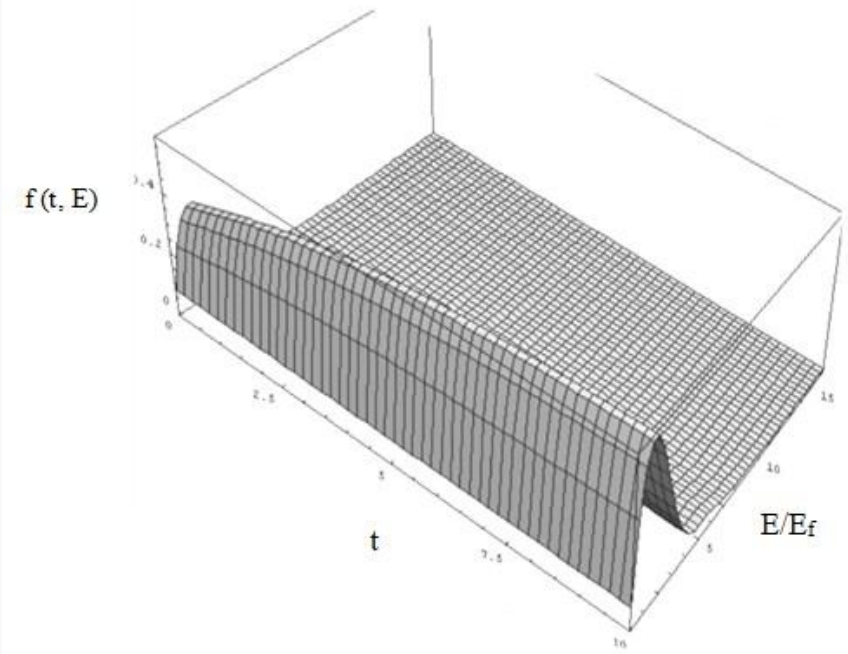

Figure 1: Evolution of time and energy distribution function

Under the impact of laser EMR, a non-equilibrium state of the electronic subsystem occurs in a solid-state medium, which leads to changing its electrophysical characteristics. According to the results of known works by Karas V.I., Novikov V.E., Konyakhin H.F., Sotnikov A.M., the distribution function becomes two-component, and the dielectric constant becomes complex.

The dependence of the imaginary part of the dielectric constant of the media on the phase velocity of the laser EMR and the ratio of the densities of the non-equilibrium and equilibrium parts of the distribution function is shown in fig. 2 .

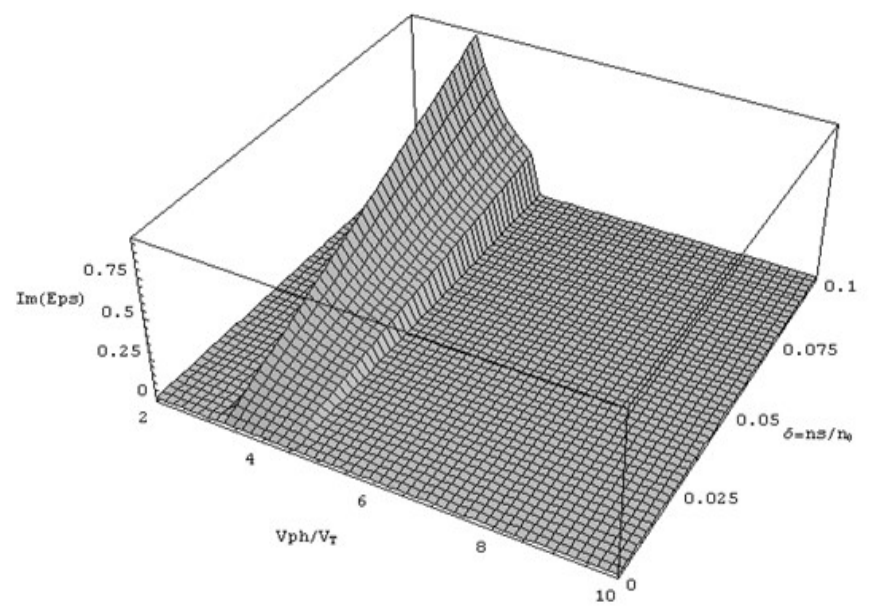

Figure 2: The dependence of the imaginary part of the dielectric constant of the media on the phase velocity of the laser EMR and the ration of the densities of the non-equilibrium and equilibrium parts of the distribution function 
The results of numerical calculations of the imaginary part of the dielectric constant of the medium from the phase velocity of the laser EMR point to the existence of an area in which the imaginary part is insignificant, which, in its turn, indicates the possibility of propagation of the laser EMR in these areas of phase space, but at the same time there is a significant variation in the dispersion properties that occur in the equilibrium state, which causes the possibility of reflection of the laser EMR from the solid-state plasma media.

The surface on which the results of the numerical calculation of the dependence of the laser EMR frequency on the phase velocity and the ratio of densities on the non-equilibrium and equilibrium parts of the distribution function are presented, is shown in fig. 3.

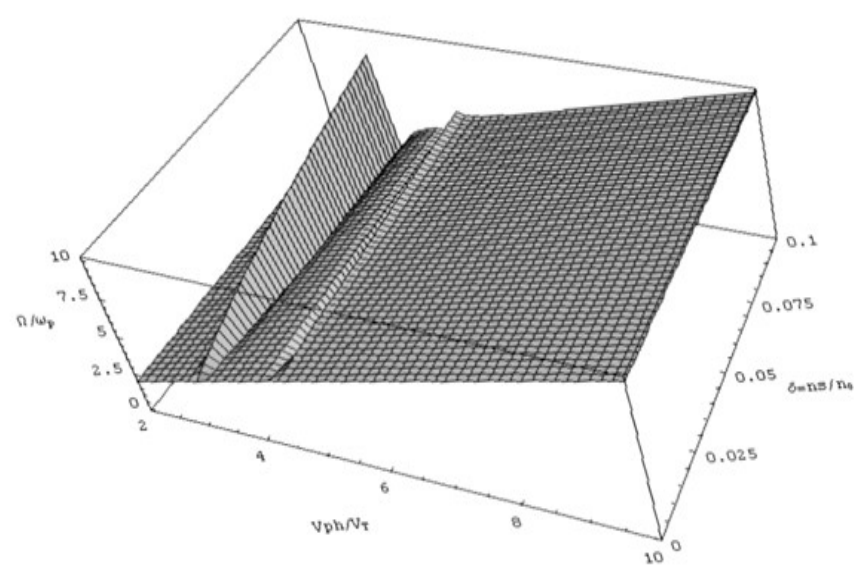

Figure 3: The dependence of the laser EMR frequency on the phase velocity and the ratio of densities on the non-equilibrium and equilibrium parts of the distribution function

The dispersion of longitudinal waves at $\varepsilon(\omega, \mathrm{k})=0)$ can be represented by the expression [3]:

$$
\omega^{2}=\frac{\omega_{\mathrm{p}}^{2}}{1+\frac{\omega_{\mathrm{ps}}^{2}}{\mathrm{k}^{2} \mathrm{v}_{\mathrm{i}}^{2}}} .
$$

$\omega_{\mathrm{ps}}-$ the frequency of acoustic plasma waves;

$v_{i}$ - the velocity of electronic sound;

$\mathrm{k}$ is a wave vector.

The expression (1) can be presented in the following way:

$$
\Omega_{\mathrm{p}}^{2}=\frac{1}{1+\frac{\mathrm{k}_{\mathrm{ds}}^{2}}{\mathrm{k}^{2}}},
$$

where $\Omega_{\mathrm{p}}^{2}=\frac{\omega^{2}}{\omega_{\mathrm{ps}}^{2}}$.
For condition $\mathrm{k} \ll \mathrm{k}_{\mathrm{ds}}$, для $\mathrm{W}=5 \mathrm{eV}$ according to the expression y $_{\mathrm{i}} \approx 6 \cdot 10^{7} \sqrt{\mathrm{W}} \mathrm{sm} / \mathrm{s}$, let us calculate the dependence of the laser EMR frequency on the wave number and the ratio of the densities of the non-equilibrium and equilibrium parts of the distribution function.

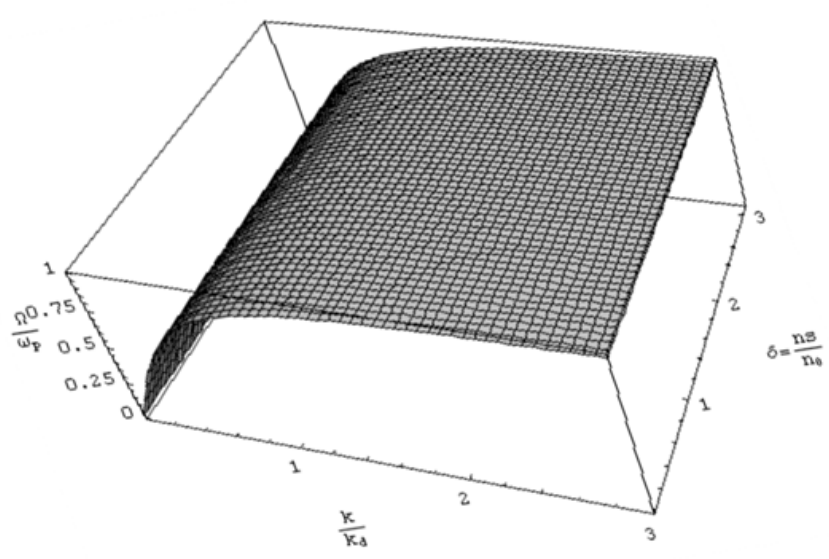

Figure 4: The surface that characterizes the dependence of the laser EMR frequency on the wave number and the ration of the densities of the non-equilibrium and equilibrium parts of the distribution function.

The analysis of the dependence of the laser EMR frequency on the wave number and the ratio of the densities of the non-equilibrium and equilibrium parts of the distribution function, which is presented in fig. 4 , shows that there is a wide area in which, under the influence of the laser EMR, its frequency becomes close of the frequency of plasma oscillations, which indicates the reflection of the laser EMR from solid-state plasma material. In the conditions of decay instability, the dispersion equation (1) for determining the threshold and the growth increment has the following form:

$$
\begin{aligned}
\mathrm{v}_{\mathrm{i}} & \approx 6 \cdot 10^{7} \sqrt{\mathrm{W}} \mathrm{k}^{2} \mathrm{r}_{\mathrm{d}}^{2}+\frac{\omega^{2}}{\omega^{2}-\omega_{\mathrm{ps}}^{2}}-\mathrm{i}\left[\mathrm{k}^{4} \mathrm{r}_{\mathrm{d}}^{4} \delta \varepsilon_{\mathrm{e}}^{\prime \prime}(\omega, \mathrm{k})+\frac{\omega^{4}}{\left(\omega^{2}-\omega_{\mathrm{ps}}^{2}\right)} \delta \varepsilon_{\mathrm{i}}^{\prime \prime}(\omega, \mathrm{k})\right]= \\
& =\frac{\mathrm{k}^{2}}{4}\left\{\frac{\left(\mathbf{k}-\mathbf{k}_{\mathbf{0}} \mathrm{v}\right)^{2}}{\left(\mathbf{k}-\mathbf{k}_{\mathbf{0}}\right)^{2}}\right\} \frac{1}{2 \omega \omega_{0}-\mathrm{c}^{2}\left(\mathbf{k}^{2}-2 \mathbf{k} \mathbf{k}_{\mathbf{0}}\right)+\mathrm{i} \omega_{0}^{2} \varepsilon_{\mathrm{e}}^{\mathrm{tr}}\left(\omega_{0}\right)}- \\
& -\frac{\left(\mathbf{k}+\mathbf{k}_{\mathbf{0}} \mathrm{v}\right)^{2}}{\left(\mathbf{k}+\mathbf{k}_{\mathbf{0}}\right)^{2}} \cdot \frac{1}{2 \omega \omega_{0}-\mathrm{c}^{2}\left(\mathbf{k}^{2}+2 \mathbf{k k}_{\mathbf{0}}\right)+\mathrm{i} \omega_{0}^{2} \varepsilon_{\mathrm{e}}^{\mathrm{tr}^{\prime \prime}}\left(\omega_{0}\right)},
\end{aligned}
$$

where $r_{d}$ is the Debye radius;

$\mathrm{k}_{0}$ - the wave number of the laser EMR.

According to the expression (4), we will write the ratio for the instability increment in the following form $[2,9]$ : 
Maksym Iasechko et al., International Journal of Emerging Trends in Engineering Research, 8(2), February 2020, $568-573$

$$
\gamma_{\mathrm{H}}(\mathrm{k})=\frac{\omega_{\mathrm{p}}}{32 \pi} \frac{\mathrm{a}_{0}^{2}}{\mathrm{n}_{0} \mathrm{~T}_{\mathrm{e}}}\left(\frac{\mathrm{k}}{\mathrm{k}_{\mathrm{ds}}}\right)^{2} \operatorname{Im} \varepsilon(\omega, \mathrm{k}),
$$

where $\alpha_{0}$ is the amplitude of Langmuir waves;

$\mathrm{n}_{0}$ is the initial electron density in the solid-state plasma material;

$T_{e}$ is the temperature of the electrons of the solid-state plasma material.

The use of the mechanism of decay instability leads to an increase in the magnitude of the reflection energy of the laser EMR.

The expression to determine the increment of the amplitude increase makes it possible to estimate the increase in the reflection coefficient of the laser EMR from the solid-state plasma medium in the non-equilibrium state compared to the equilibrium one. To do this, we will use the ration of the corresponding parameters, taking into account the values of the wave numbers $\mathrm{k}$ and $\mathrm{k}_{\mathrm{ds}}$ :

$$
\frac{\gamma_{\mathrm{H}}(\mathrm{k})}{\gamma_{0}(\mathrm{k})}=\left(\frac{\omega_{\mathrm{p}}}{\mathrm{V}_{\mathrm{TE}}} \frac{\mathrm{v}_{\mathrm{i}}}{\omega_{\mathrm{ps}}}\right)^{2}
$$

For conditions $\mathrm{W}=5 \mathrm{eV}, \omega_{\mathrm{ps}}=3 \cdot 10^{13} \mathrm{~Hz}, \mathrm{~V}_{\mathrm{TE}} \approx 10^{6} \mathrm{sm} / \mathrm{s}$, according to the expression $\mathrm{v}_{\mathrm{i}} \approx 6 \cdot 10^{7} \sqrt{\mathrm{W}}, \mathrm{sm} / \mathrm{s}$, let us calculate the ratio of the growth increments:

$$
\frac{\gamma_{\mathrm{H}}(\mathrm{k})}{\gamma_{0}(\mathrm{k})} \approx(1 \ldots 2) \cdot 10^{3} \ldots 10^{4}
$$

The surface that characterizes the dependence of the ratio of the amplitude increase increments depending on the phase velocity of the laser EMR and the ratio of the densities of the non-equilibrium and equilibrium parts of the distribution function is provided in fig. 5 .

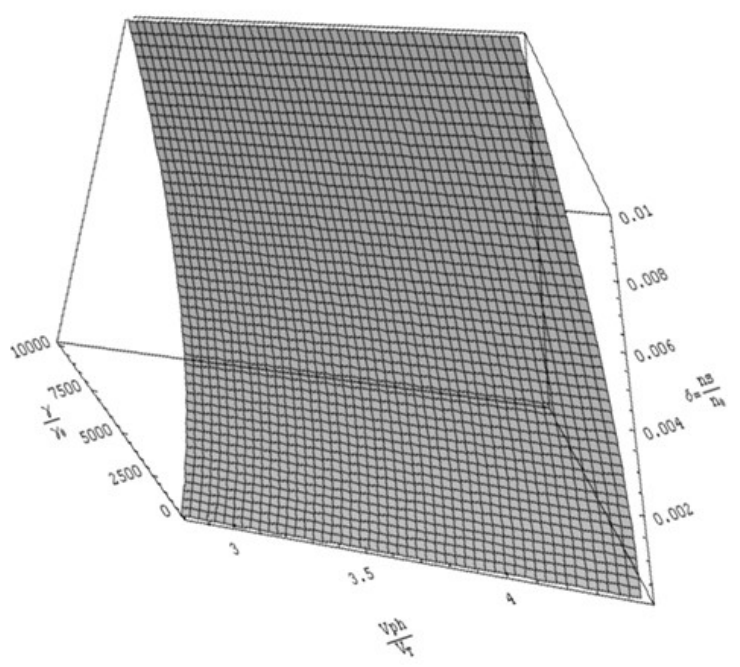

Figure 5: The dependence of the ratio of the amplitude increase increments depending on the phase velocity of the laser EMR and the ratio of the densities of the non-equilibrium and equilibrium parts of the distribution function

Thus, the creation of decay instability conditions in a solid-state plasma medium ensures the increase in the laser EMR reflection up to $10^{3} \ldots 2 \cdot 10^{4}$ times at a small thickness of the shielding material.

\section{CONCLUSION}

The results of the study of the shielding properties of the solid-state plasma medium with hexaferrite elements for the REM protection against laser EMR radiation have been presented in the article.

The impact of decay instability on the dispersion properties of the solid-state plasma media and the reflection of laser radiation have been estimated. It has been shown that the creation of decay instability conditions in the solid-state plasma medium ensures the increase in the laser EMR reflection up to $10^{3} \ldots 2 \cdot 10^{4}$ times at a small thickness of the shielding material.

\section{REFERENCES}

1 M. Iasechko. Plasma technologies for the protection of radio electronic means from exposure to high-power electromagnetic radiations with ultrashort pulse duration, Proceedings of the 1-st Annual Conference, Tallinn, Estonia, 2017, pp. 18-21. doi: /10.21303/2585-6847.2017.00480.

2 E. M. Bazelyan and U. P. Raizer. Lightning attraction mechanism and the problem of laser lightning control, Physics-Uspekhi, 43:7, Moscow, 2000, pp. 701-716.

3 V. L. Ginzburg and A. V. Gurevich. Nonlinear phenomena in a plasma located in an alternating electromagnetic field, SOV PHYS USPEKHI, 3(1), 
Moscow, doi:10.1070/PU1960v003n01ABEH003261.

4 O. G. Sytenko. Electromagnetic plasma fluctuations, KhGPU, Ukraine, Kharkiv, 1965, pp. 1-183.

5 S.A.Gutsev, A.A. Kudryavtsev, R.Yu. Zamchiy, V.I. Demidov, and V.I. Kolobov. Diagnostics and modeling of ashort (without positive column) glow discharge in helium with nonlocal plasma, Proc. 40th European Physical Society Conference on Plasma Physics, Finland, 2013, N 06.502.

6 M.M. Iasechko, and O.M. Sotnikov. Advanced technologies of radio electronic equipment (means) protection from powerful electromagnetic radiations with ultra short duration of pulses exposure, Published by Izdevnieciba Baltija Publishing, Collective monograph, Riga, 2018, pp.356-385.

7 I. Mac-Daniel. Collision processes in ionized gases, World, Moscow, 1967.

8 O.Skoblikov and V. Knyazyev. Properties of Conductive Shells Exposed to Electromagnetic Impulse of Lightning, International Conference on Lightning Protection (ICLP'2012), Vienna, Austrian, 2012, pp. 1-8.

9 A. Tajirov, I. Cwhanovskaya, and Z. Barsova, N. Iluoykha. Chemistry and technology of magnetite and barium-containing composite materials on its basis, European Science and Technology: materials of the II international research and practice conference, Wiesbaden Germany, 2012, pp. 80-87.

10 V.A. Chernikov, S.A. Dvinin, A.P. Ershov, I.B.Timofeev, and V.M. Shibkov. Experimental and Theoretical research of DC transversal gas discharge in a supersonic gas flow, The 3rd workshop on Magneto-Plasma-Aerodynamics in Aerospace Applications, Moscow, 2001, pp. 129-134.

11 B. M. Smyrnov. Low Ionized Gas Physics, The science, Moscow, 1985.

12 S.A. Dvinin, and A.A. Kuzovnikov. Plane ionization waves caused by diffusion in high frequency fields, XVII International Conference on Phenomena in Ionized Gases, Belgrade, 1989, pp. 818-819.

13 O. Sotnikov, M. Iasechko, V. Larin, O. Ochkurenko, and D.Maksiuta. The model of a medium for creation of electric hermetic screens of the radio electronic means, IJATCSE. 8(2), 2019, pp. 300-304. doi:10.30534/IJATCSE/2019/32822019.

14 M. Iasechko, O. Tymochko, Y. Shapran, I. Trofymenko, D. Maksiuta, and Y. Sytnyk. Loss definition of charged particles in the discharge gap of the opening of the box-screens during the formation of a highly conductive channel, IJATCSE. 8(1.3), 2019, pp. 1-9. doi: 10.30534/ijatcse/2019/0181.32019.

15 M. Iasechko, V. Larin, O. Ochkurenko, S. Salkutsan, L. Mikhailova, and O. Kozak. Formalized Model Descriptions Of Modified Solid-State Plasma-Like Materials To Protect Radio-Electronic Means From The Effects Of Electromagnetic Radiation, IJATCSE. 8(3), 2019, pp. 393-398. doi: 10.30534/ijatcse/2019/09832019.

16 M. Iasechko, V. Larin, O. Ochkurenko, A. Trystan, T.Voichenko, A. Trofymenko, and O. Sharabaiko.
Determining the function of splitting the charged particles of the strongly ionized air environment in the openings of the case-screens of radioelectronic means, IJATCSE. 8(1.3), 2019, pp. 19-23. doi: 10.30534/ijatcse/2019/0481.32019.

17 M.M. Iasechko, and O.M. Sotnikov. Protecting of radio electronic facilities is from influence of powerful electromagnetic radiation, Published by Izdevnieciba Baltija Publishing, Collective monograph, Riga, 2019, pp.283-299.

18 A. Syrotenko, O. Sotnikov M. Iasechko, V. Larin, S.Iasechko O. Ochkurenko, and A. Volkov. Model of Combined Solid Plasma Material for the Protection of Radio-Electronic Means of Optical and Radio Radiation, IJATCSE, 8(4), 2019, pp. 1241 - 1247. doi:10.30534/ijatcse/2019/33842019.

19 V. Gurevich. Electromagnetic Terrorism: New Hazards. - Electrical Engineering and Electromechanics, N 4, 2005.

$20 \mathrm{Yu}$. P. Reiser. Breakdown and heating of gases under the action of laser beam, The successes of the physical sciences, 1965.

21 A.V. Bobylev, and V.A. Chuyanov. On the numerical solution of the Landau kinetic equation, Journal of Computational Mathematics and Mathematical Physics, $T$. 16. № 2, 1977, pp. $407-416$.

22 O. Turinskyi, M. Burdin, M. Iasechko, V. Larin, Y. Gnusov, D. Ikaev, V. Borysenko, and V. Manoylo. Protection of board radioelectronic equipment from the destructive powerful electromagnetic radiation with the use of natural technologies, IJETER, 7(11), 2019, pp. 542 — 548. doi: 10.30534/ijeter/2019/237112019.

23 M. Iasechko, V. Larin, D. Maksiuta, O. Ochkurenko, I. Krasnoshapka, Y.Samsonov, H. Lyashenko, A.Zinchenko, and R.Vozniak. Model description of the modified solid state plasma material for electromagnetic radiation protection, IJETER, 7(10), 2019, pp. 376 - 382. doi: 10.30534/ijeter/2019/027102019.

24 O. Turinskyi, M. Iasechko, V. Larin, D. Dulenko, V. Kravchenko, O. Golubenko, D.Sorokin, and O. Zolotukhin. Model and development of plasma technology for the protection ofradio-electronic means of laser emission, IJATCSE. 8(5), 2019, pp. 2429-2433. doi:10.30534/IJATCSE/2019/85852019.

25 M.Iasechko, Y. Gnusov, I. Manzhai, O. Uhrovetskyi, V.Manoylo, A. Iesipov,O. Zaitsev, M. Volk, and O. Vovk. Determination of requirements for the protection of radio-electronicequipment from the terroristic influence by electromagnetic radiation, IJETER, 7(12), 2019, pp. 772 - 777. doi: 10.30534/ijeter/2019/077122019.

26 H. Khudov, A. Zvonko, S. Kovalevskyi, V. Lishchenko, and F. Zots. Method for the detection of smallsized air objects by observational radars, Eastern-European Journal of Enterprise Technologies, № 2/9 (92), 2018, pp. 61-68. DOI: 10.15587/1729-4061.2018.126509.

27 I.Ruban, H. Khudov, O.Makoveichuk, M. Chomik, V.Khudov, I. Khizhnyak, V.Podlipaiev, Y.Sheviakov, O.Baranik, and A.Irkha. Construction of methods for contours of objects on tonal aerospace images based on the ant aigoritms, Eastern-European Journal of Enterprise 
Maksym Iasechko et al., International Journal of Emerging Trends in Engineering Research, 8(2), February 2020, 568- 573

Technologies, № 5/9(101), 2019, pp.25-34. doi: 10.15587/1729-4061.2019.177817.

28 M.Iasechko, M. Mozhaiev, I. Manzhai, M. Volk, V. Manoylo, O. Ochkurenko, D. Maksiuta, V. Larin, A. Markov, and O. Kostyria. Conditions for reliable transmission of information over long distancesusing a powerful electromagnetic radiation, IJETER, 8(1), 2020, pp. 138 - 144. doi: 10.30534/ijeter/2020/18812020.

30 M. Iasechko, V. Larin, D. Maksiuta, O. Ochkurenko, I. Krasnoshapka, Y.Samsonov, H. Lyashenko, A.Zinchenko, and R.Vozniak. Model description of the modified solid state plasma material for electromagnetic radiation protection, APRN Journal of Engineering and Applied Sciences, 14(20), 2019, pp. 3566 - 3571. 